\title{
Research on the Applications of Electronic Information Technology in Intelligent Traffic Light Signal Control
}

\author{
Juan Zhong ${ }^{1, *}$, Hua Liao ${ }^{2}$ \\ ${ }^{1}$ College of Information Engineering, Gannan Medical University, Ganzhou, 341000, China \\ ${ }^{2}$ STATE GRID GANZHOU ELECTRIC POWER SUPPLY COMPANY, Ganzhou, 341000, China \\ *Corresponding Author: Juan Zhong
}

Keywords: Electronic information technology, Intelligent traffic light signal control, Intelligent transport

\begin{abstract}
With the rapid development of urbanization in China, the problem of traffic congestion is becoming more and more serious. The development of an intelligent traffic signal control system to alleviate traffic congestion is a very valuable research subject. In this paper, the functional requirements of intelligent traffic signal control system are discussed, and the design scheme of intelligent control system of the traffic light is given on the basis of electronic information technology to provide some references for the relevant researchers.
\end{abstract}

\section{Introduction}

At present, the car gradually goes into people's lives. While it brings convenience to people's life, it also brings traffic jams and even frequent traffic accidents. Therefore, in the fast-paced life of the city, the traffic signal lights to improve traffic conditions play a key role. The relevant departments of our governments require us to actively study the traffic rules according to the actual situation of the adjustment interval of intelligent traffic lights and traffic jams. The application of electronic information technology can greatly reduce the cost of hardware, but also improve flexibility, effectively improve the traffic order. Along with the city to speed up the process, more traffic congestion phenomenon is serious. When the road began to worse, more and more accidents occur caused by traffic problems. The traffic problem has become one of the most serious social problems. The deterioration of traffic order has a certain impediment to the process of urbanization and influences the construction and development of modern cities. Therefore, it is important for traffic system to improve traffic order and formulate corresponding rules to alleviate traffic congestion and improve traffic rate of important traffic intersection. In the management of the whole traffic system, the traffic signal control is the most important control element. The traffic signal is very important, and it will play a big role in improving the traffic rate.

The development of intelligent transportation is inseparable from the development of Internet of things. The continuous development of the concept of Internet of things technology can make the intelligent transportation system become more and more perfect. Intelligent transportation is the embodiment of traffic combination. The twenty-first century will be the century of highway traffic intelligent. The intelligent transportation system is a kind of advanced integrated traffic management system. In this system, the vehicle is free on the road by their own intelligence and the road adjust to the best state on its own intelligent traffic flow. With the aid of the system, the managers have good command of the road and vehicles. Its prominent feature is that the intelligent transportation system takes the information collection, processing, publishing, analysis and utilization as the main line to provide traffic participants with a variety of services.

With the development of science and technology, virtual instrument technology has been widely used, especially in the control of automatic testing, data acquisition and instrument quality. The use of these technologies can promote the realization of the design of the measurement and control 
greatly. In this paper, the importance of the application of electronic information technology in traffic signal control is analyzed, and its applications are specifically described.

\section{Functional Requirements of Intelligent Traffic Signal Light Control System}

Traffic light is the core of traffic control. It can effectively command the communication between vehicles and pedestrians. The intelligent traffic lights system has three colors, namely red, yellow, and green. The red light is on behalf of the prohibition of passing. The green light is on behalf of access and while the yellow light plays a warning role. Intelligent traffic signal lamp system can effectively enhance the safety of road traffic and the traffic order. At the same time, using the intelligent traffic signal lamp system can improve the road rate, reduce the probability of safety accidents, alleviate traffic conditions. The traffic lights are set up is reasonable. Not all junctions will set up lights, instead of concentrated flow and large traffic crossroads settings. It can effectively improve the road utilization rate, reduce the probability of occurrence of accidents. The vehicle acceptance system is installed inside the vehicle and completes the traffic command through close connection within the vehicle system.

The effective guarantee is conducive to better implementation of road traffic management, reducing the incidence of road traffic accidents, improving the use efficiency of roads and easing traffic conditions. In the road traffic network, not all are set intersection traffic lights. But in the stream of people, relatively dense traffic crossroads, intersection, traffic lights transform signal, it is controlled by the machine. In the indicative function of traffic signals and other signals, vehicle and pedestrian pass orderly. In the vehicle receiving device, there are four units, which are receiving unit, control unit, voice unit and display unit.

When the intelligent traffic signal lamp system works, the signal message is firstly displayed by the traffic signal lamp system. The on-board device receives the signal of the transmitting device and receives the receiver effectively. After decoding, the on-board device transmits the signal to the language unit or sends it to the display unit. There is a very close connection among the four units, and they are indispensable.

\section{Relationship between Intelligent Control System of Traffic Signal Light and Electronic Information Technology}

With the emergence of intelligent traffic lights control system, it provides the possibility to solve these problems. The intelligent algorithm is used to solve the traffic control problem. Electronic information technology has its own unique advantages, for special instrument system. The user can customize the electronic, electronic information technology. The function is flexible, simple constructed and more and more widely applied to various fields, especially in the field of scientific research, development, measurement and detection. It plays an irreplaceable important role. Internationally, hardware is becoming more and more software in the process of development, showing the characteristics of hardware and software, and the advanced nature of electronic information technology coincides with the international trend. The virtual instrument technology of electronic information technology has very powerful function based on ordinary instruments, logical analysis, signal filtering and other functions can be achieved, at the same time, this technology, also equipped with a special probe and detection of specific software, the system parameters can be effectively realized, such as automobile engine parameter detection, furnace temperature detection. The operation of the virtual instrument technology is very high, fully graphical, minimalist style user-friendly. It can not only high-speed data acquisition equipment automatic measurement system, at the same time, but also can control equipment, integrated automatic control system. Three colors of the signal lights can be alternately lit in the intelligent light traffic control system through the application of electronic information technology. It reduces the incidence of road traffic accidents. At the same time, the traffic lights can be monitored in real time. 


\section{Designs of Intelligent Control System of Traffic Signal Light Based on Electronic Information Technology}

Overall Design. Intelligent traffic signal light is to ensure the safety of people and vehicles. At the crossroads, we should set up four traffic signal lights in different ways to ensure traffic safety operation. When the east and west directions are green, the red light rises in the north and south, and the yellow light is over the stage. On the contrary, when things turn red, the north and south directions are green. In the design of intelligent traffic signal lamp, we should give full consideration to the actual situation. In daytime, traffic congestion should be appropriately arranged to reduce the time interval of the traffic light to prevent traffic jams. At night, the vehicles and pedestrians should be less, appropriate to extend the interval time of traffic lights. At the same time, when traffic lights are working, the working status should be given back to the program control terminal in time, so that it can be monitored in real time.

When the red light in one direction is turned on, the cross direction corresponds to the green light, and the yellow light appears at the transition stage, and vice versa. The traffic is different because of the traffic at the intersection. Traffic is busy during the day, traffic lights change more quickly, so as to improve traffic efficiency and reduce congestion; on the contrary, when traffic is sparse at night, traffic lights need to change slowly. This function can be achieved by the control program by changing the duration of the signal status. In addition, through the program and simple peripherals can feedback to the control terminal signal lamp working condition, according to the state level feedback information to determine the traffic lights in the process of switching is abnormal, and then realize the working state of real-time monitoring of traffic lights function.

The design should fully consider the above factors, we should take the traffic control terminal, traffic power management, data acquisition, traffic control center four link, to ensure that the intelligent traffic signal system can work normally. On the basis of traffic congestion prediction, an intelligent traffic signal control system based on information fusion is designed. The system should include vehicle flow forecasting module, traffic jam prediction module and signal control module. The traffic flow prediction module carries out the wavelet neural network prediction through the traffic flow data collected by the video monitor on the road. The traffic congestion prediction module integrates the traffic flow information and the vehicle speed information through the cusp catastrophe theory, and obtains the critical traffic flow value when the traffic congestion occurs. The signal control module is integrated the first two module to obtain a more reasonable scheduling strategy.

Design of Control Terminal Module. Most of the traffic lights in the road are fixed periodic signals. The serious disadvantage of this kind of signal controller is that it can only change the signal cycle according to the set period of change. It is very likely that a large number of vehicles are waiting for traffic, while vehicles in the direction of traffic are no longer waiting for traffic.

Such intersections often waste road resources, and traffic congestion is easy to occur. Intelligent traffic control terminal module is the concentrated embodiment of the application of electronic information technology in intelligent traffic lights. In the traffic control terminal module, we should pay attention to the change of cascading sequential structure. In the design of traffic control terminal module, we should consider from many aspects, and can make the signal light alternately through reasonable sequence structure. The designers have to consider that the lights need to last for some time.

Therefore, the use of electronic information technology uses the default timing data as a reference. Through the corresponding settings, yellow lights are joined into warning system between the people and vehicles. The intelligent traffic control terminal module is the concentrated embodiment of the application of the electronic information technology in the intelligent traffic signal light system. The traffic control terminal module is responsible for controlling the color changes of the traffic lights and the duration of the lights. The control module transmits the incoming control information to the computer. After corresponding processing, the computer is returned to the control terminal, and the control terminal carries out intelligent control of the traffic signal lamp according to the instruction requirements. 
The design of the intelligent traffic control terminal adopts a flexible, high reliability and cascading sequence framework, which is under the edit state. The order of each frame structure can be easily changed. The system set up two different directions of traffic lights. Therefore, it needs to use two sequential structure as the traffic lights switch colors. It can be achieved by setting local variables. For each color, the duration of lighting is controlled by the timer provided by the system tool. It is implemented by parameter setting. When the signal light is near the end of the system, the system needs to remind the vehicle and pedestrians to pay attention to safety. We have finally completed the control terminal module design of intelligent traffic light signal control system based on electronic information technology.

Design of Control Center Module. The module is mainly designed by logic circuit to transfer the data collected from data acquisition module to the central module. The logic circuit of the central module determines whether the traffic light is working normally and whether the circuit is faulty or not. Electronic information technology penetrates every aspect of intelligent traffic signal system, the most obvious is that intelligent traffic control center module, intelligent traffic control center module is mainly to judge in the received information, so as to realize the fault of traffic lights. The use of signal coordination control depends largely on the arrival characteristics of the traffic flow at the intersection. The traffic was blocked during the red-light crossing, and the traffic began to flow at saturation light. At the intersection exit, traffic shows a queue pattern and runs down the road toward the downstream intersection.

When traffic reaches downstream intersection, we can maintain the queue and then take signal coordination, adjust the green good starting time of downstream intersections. The team can significantly reduce the traffic vehicle delay queue. The module of traffic control center contains logic circuit, which can accurately judge the information transmitted by data acquisition module, monitor the working state of traffic signal in real time, and ensure the normal operation of traffic. Intelligent traffic signal lamp system is in normal working condition will shine a light red, yellow, green, if the signal lamp system is in this state when the fault occurs. The intelligent traffic control center module will timely alert the staff to make the fastest response. After the data acquisition module completes the information collection, it needs to transfer the information to the central module. The transmission of information is realized by logical circuit design. After the central module receives the information, the logical circuit judges the information, and understands the real-time working state of the traffic light to ensure the normal operation of the traffic light. It can handle the fault in a timely manner. When the traffic lights are in normal condition, the signal will only light red, green and yellow light one after another. If it is a fault, the system will alarm. Under the action of the warning light, the staff can handle the fault in time to ensure traffic safety.

\section{Conclusion}

With the rapid development of urban traffic, the construction and application of intelligent traffic signal light system is becoming more and more important and indispensable. The introduction of electronic information technology in the control of intelligent traffic signal lights further improves the role of the signal system in the road traffic system. Therefore, with the continuous development of electronic information technology, intelligent traffic light signal control system should actively introduce electronic information technology to guarantee the stable operation of traffic.

\section{References}

[1] Jin Zhi. Design of intelligent traffic light control system based on electronic license plate [J]. Wireless Internet Technology, 2017(7): 54-55.

[2] Zhou Wenqi, Han Xiaoyu. Study on the application of electronic information technology in intelligent traffic signal control [J]. Electronic Test, 2015(21): 91-92. 
[3] Liu Daoqun. Discussion on A pplication of Information Technology in New Generation of Intelligent Transport System [J]. Computer Science, 2014, 41(11A): 55-56+68.

[4] Zhang Lizhe, Qiu Xin, Zhang Fusheng. Research on Intelligent Transportation Information Service System Based on Mobile Communication Technology [J]. Mobile Communications, 2015(18): 82-86. 